\title{
EXPANSION PROBLEMS IN CONNECTION WITH HOMOGENEOUS LINEAR $q$-DIFFERENCE EQUATIONS*
}

\author{
BY \\ M. G. CARMAN
}

I. INTRODUCTION

1. Statement of the problem. This paper has for its purpose the development of expansion theories for the general homogeneous linear $q$ difference equation with analytic coefficients analogous to those of Neumann and Gegenbauer for Bessel's equation. $\dagger$

In Part II is treated the expansion of an arbitrary analytic function of a single variable in series analogous to those of Maclaurin and Laurent. In Part III expansions of functions of several variables are considered.

II. Expansion of ANALYTIC FUNCTIONS OF A SINGLE VARIAble

2. Solutions of the $q$-difference equation. In the equation

$$
\begin{aligned}
A_{0}(x) u_{n}\left(q^{k} x\right)+A_{1}(x) u_{n}\left(q^{k-1} x\right)+\cdots & +A_{k-1}(x) u_{n}(q x) \\
& +\left(\lambda_{n}+A_{k}(x)\right) u_{n}(x)=0
\end{aligned}
$$

we shall suppose that $q$ is a constant greater than unity in absolute value, that $k$ is a fixed positive integer, that $n$ is a non-negative integral parameter, and that the coefficients $A_{0}(x), A_{1}(x), \cdots, A_{k}(x)$ are analytic functions of $x$ at $x=0, A_{0}(0)$ being different from zero. Let $R$ be the absolute value of the singularity nearest $x=0$ of any function of the set $1 / A_{0}(x), A_{0}(x)$, $A_{1}(x), \cdots, A_{k}(x)$ and for $|x|<R$ let $A_{i}(x)$ have the expansion

$$
A_{i}(x)=\sum_{j=0}^{\infty} a_{i j} x^{i} \quad(i=0,1,2, \cdots, k) .
$$

The parameter $\lambda_{n}$ shall be so determined that equation (1) has a solution of the form

$$
u_{n}(x)=x^{n+\nu}\left(1+\sum_{s=1}^{\infty} c_{n s} x^{s}\right)
$$

* Presented to the Society, May 1, 1926; received by the editors in December, 1925.

† See Watson, Theory of Bessel Functions, London, 1922, where these results are summarized. 
where $\nu$ is any constant. To see that this is possible, substitute this series for $u_{n}(x)$ in (1), and equate to zero the coefficients of the various powers of $x$. This formal procedure gives the results

$$
a_{00} q^{k(n+\nu)}+a_{10} q^{(k-1)(n+\nu)}+\cdots+a_{k 0}+\lambda_{n}=0
$$

and

$$
c_{n s}\left(\lambda_{n}-\lambda_{n+s}\right)=\sum_{i=0}^{s-1} c_{n i} S_{n, s-i}^{(i)} \quad(s=1, \tilde{L}, 3, \cdots)
$$

where

$$
S_{n r}^{(i)}=-\sum_{j=0}^{k} a_{j r} q^{(k-j)(n++i)}
$$

and $c_{n 0}$ is to be taken equal to unity.

Equation (4) will be taken as the definition of $\lambda_{n}{ }^{*}$ By (5) the coefficients $c_{n s}$ are uniquely determined, provided that the relation

$$
\lambda_{n}-\lambda_{n+s} \neq 0
$$

obtains for all non-negative integers $n$ and all positive integers $s$. By forming the difference $\lambda_{n}-\lambda_{n+s}$, it is easy to see that as either $n$ or $s$ becomes infinite or as both become infinite simultaneously the absolute value of this difference becomes infinite. Consequently relation (6) holds for all except at most a finite number of pairs of values of $n$ and $s$. It will be supposed that the quantities $q, k, \nu, a_{00}, a_{10}, \cdots, a_{k 0}$ are such that (6) holds for this finite number also.

It will now be shown that, with the $c_{n s}$ determined by (5), the series for $u_{n}(x)$ in (3) is convergent $\dagger$ for $|x|<|q|^{k} R$ and represents a solution of (1) for $|x|<R$. Since the expansions (2) are valid for $|x|<R$, a constant $a$ exists such that

$$
\left|a_{i j}\right|<a^{j}
$$

*If instead of equation (1) we write

$$
A_{0}(x) u_{n}\left(q^{k} x\right)+A_{1}(x) u_{n}\left(q^{k-1} x\right)+\cdots+A_{k}(x) u_{n}(x)+\lambda_{n} u_{n}\left(q^{h} x\right)=0,
$$

where $h$ is a non-negative integer not greater than $k$, equation (4) becomes

while equation (5) becomes

$$
a_{00} q^{k(n+\nu)}+a_{10} q^{(k-1)(n+\nu)}+\cdots+a_{k_{0}}+\lambda_{n} q^{h(n+\nu)}=0,
$$

Using instead of (8) the relation

$$
c_{n 8} q^{h(n+8+\nu)}\left(\lambda_{n}-\lambda_{n+8}\right)=\sum_{i=0}^{8} c_{n i}^{1} S_{n, 8-i}^{(i)}
$$

$$
\left|q^{-(k-h)(8+n)}\left(\lambda_{n}-\lambda_{n+8}\right)\right|>1 / M
$$

the convergence of the series corresponding to (3) follows just as it does when $h=0$.

$\dagger$ If the real part of $n+\nu$ is negative the terms of the series are not defined for $x=0$. The series multiplied by $x^{-\nu}$, however, is convergent in this case. 
for every $i$ and $j$. Consequently there is a constant $A$ such that

$$
\left|S^{(8-r)} q^{-k(8+n)}\right|<A^{r}
$$

for every $s, n$, and $r$. Also $q^{-k(s+n)}\left(\lambda_{n}-\lambda_{n+s}\right)$ is bounded away from zero as either $n$ or $s$ becomes infinite or as both become infinite simultaneously, so that by virtue of (6) we may infer the existence of an $M>1$ such that

$$
\left|q^{-k(s+n)}\left(\lambda_{n}-\lambda_{n+s}\right)\right|>1 / M
$$

for every $s$ and $n$. Using the bounds (7) and (8) we have by induction from (5) that

$$
\left|c_{n s}\right|<2^{s-1} M^{s} A^{s}
$$

or, putting $K=2 M A$,

$$
\left|c_{n s}\right|<K^{s}
$$

for every $s$ and $n$. Hence the series (3) is absolutely convergent for $|x|>K^{-1}$. It represents a solution of (1) for $|x|<|q|^{-k} K^{-1}$ since then the operations performed in the calculation of the coefficients $c_{n s}$ are justifiable. From equation (1) one sees that the singularity nearest the point $x=0$ of any solution of (1) which is analytic at $x=0$ is at least a distance $R$ from $x=0$. We may then conclude that the series

$$
x^{n+\nu}\left(1+\sum_{s=1}^{\infty} c_{n s} x^{s}\right)
$$

is absolutely convergent ${ }^{*}$ for $|x|<|q|^{k} R$ and represents a solution of (1) for $|x|<R$. $\dagger$

3. Expansion of $x^{m}$. Let us consider the possibility of expanding $x^{m}$ in the form

$$
x^{m}=\sum_{n=m}^{\infty} b_{m n} U_{n}(x)
$$

where we have written $U_{n}(x)$ for $x^{-v} u_{n}(x)$. If we substitute for $U_{n}(x)$ its

* See the second footnote on p. 524 .

$\dagger$ The existence of this solution might have been inferred from the general existence theorem given by R. D. Carmichael in the American Journal of Mathematics, vol. 34, p. 159, where, however, a different method is used. The fact that the present method may be employed is stated in this paper. The details are set down here in order to obtain the inequality (9) which will be used later. 
power series expansion, formally interchange the orders of summation, and equate coefficients of like powers of $x$, we have

$$
\begin{aligned}
& b_{m m}=1, \\
& b_{m, m+s}+b_{m, m+s-1} c_{m+s-1,1}+b_{m, m+s-2} c_{m+s-2,2}+\cdots+b_{m m} c_{m s}=0, s>0 .
\end{aligned}
$$

Using the inequality (9) we have by induction from (11) that

$$
\left|b_{m, m+s}\right|<(2 K)^{s} \text {. }
$$

The repeated series

$$
\sum_{n=m}^{\infty} b_{m n}\left(x^{n}+\sum_{s=1}^{\infty} c_{n s} x^{n+s}\right)
$$

is therefore absolutely convergent for $|x|<1 / 2 K$. Hence, if $|x|<1 / 2 K$, the rearrangement of this series which was made in obtaining formulas (11) is justified and the expansion (10), (11) is valid.

4. Expansion of $1 /(t-x)$. The expansion

$$
1 ;(t-x)=\sum_{m=0}^{\infty} x^{m / t^{m+1}}
$$

is valid if only $|x|<|t|$. For $|x|<1 / 2 K$ we may infer from (9) and (12) the existence of $M_{1}$ and $M_{2}$ independent of $n$ such that

and

$$
\left|1+\sum_{s=1}^{\infty} c_{n s} x^{s}\right|<M_{1}
$$

$$
\left|\sum_{s=0}^{\infty} b_{n, n+s} x^{s}\right|<M_{2}
$$

evidently $M_{1}$ is independent of $x$ and $M_{2}$ is likewise if $|x| \leqq K_{1}<1 / 2 K$. Consequently the repeated series obtained from (13) by putting for $x^{\mathbf{m}}$ the expansion (10) is absolutely convergent if $|x|<|t|$ and $|x|<1 / 2 K$, for then we have

$$
\begin{aligned}
\left|\sum_{n=m}^{\infty} b_{m n} U_{n}(x)\right| & <M_{1} \sum_{n=m}^{\infty}\left|b_{m n} x^{n}\right| \\
& =M_{1}|x|^{m} \sum_{s=0}^{\infty}\left|b_{m, m+\varepsilon} x^{\bullet}\right| \\
& <M_{1} M_{2}|x|^{m} .
\end{aligned}
$$

We may then interchange the orders of summation in this repeated series and obtain the expansion

$$
1 /(t-x)=\sum_{n=0}^{\infty} v_{n}(t) U_{n}(x),
$$


where

$$
v_{n}(t)=\sum_{s=0}^{n} b_{s n} / t^{s+1}
$$

this expansion being valid for $|x|<|t|$ and $|x|<1 / 2 K$.

If $x$ is confined to the interior and boundary of a circle $c$ with center at $x=0$ whose radius is less than $1 / 2 K$ and $t$ is confined to the boundary and exterior of a circle of radius greater than that of $c$, then the convergence of the expansion is obviously uniform with respect to both $t$ and $x$.

We may now state the following theorem:

THEOREM I. There exists a constant $\sigma, 0<\sigma \leqq|q|^{k} R$, such that, if $x$ and $t$ are two complex variables satisfying the conditions

and

$$
|x| \leqq R_{1}<\sigma \text {, }
$$

$$
|t| \geqq R_{2}>R_{1}
$$

respectively, the expansion

$$
1 /(t-x)=\sum_{n=0}^{\infty} v_{n}(t) U_{n}(x)
$$

is valid and converges uniformly with regard to both $t$ and $x$.

5. Expansion of $f(x)$. Let $f(x)$ be single-valued and analytic on and within the circle $C_{2}$ of radius $R_{2} \leqq \sigma$ with center at $x=0$. Then by Cauchy's formula, for any $x$ such that $|x|<R_{2}$ we have

where

$$
\begin{aligned}
f(x) & =\frac{1}{2 \pi i} \int_{C_{2}}\left\{\sum_{n=0}^{\infty} v_{n}(t) U_{n}(x)\right\} f(t) d t \\
& =\sum_{n=0}^{\infty} a_{n} U_{n}(x)
\end{aligned}
$$

$$
a_{n}=\frac{1}{2 \pi i} \int_{C_{2}} v_{n}(t) f(t) d t
$$

This expansion converges uniformly when $|x| \leqq R_{1}<R_{2}$. We may now state

ThEOREM II. Let $f(x)$ be single-valued and analytic on and within the circle $C_{2}$ of radius $R_{2} \leqq \sigma$ with center at $x=0$. Then the expansion

$$
f(x)=\sum_{n=0}^{\infty} a_{n} U_{n}(x),
$$

where

$$
a_{n}=\frac{1}{2 \pi i} \int_{C_{2}} v_{n}(t) f(t) d t
$$


is valid if $|x|<R_{\mathbf{2}}$ and converges uniformly with respect to $x$ when $|x| \leqq R_{\mathbf{1}}<\boldsymbol{R}_{\mathbf{2}}$.

6. Relation to Maclaurin's expansion. Let $f(x)$ have the Maclaurin expansion

Then

$$
f(x)=\sum_{m=0}^{\infty} b_{m} x^{m}
$$

whence

$$
\begin{aligned}
a_{n} & =\frac{1}{2 \pi i} \int_{C_{2}} v_{n}(t)\left\{\sum_{m=0}^{\infty} b_{m} t^{m}\right\} d t \\
& =\frac{1}{2 \pi i} \sum_{m=0}^{\infty} b_{m} \int_{C_{2}} t^{m}\left\{\sum_{s=0}^{n} b_{s n} / t^{s+1}\right\} d t
\end{aligned}
$$

$$
a_{n}=\sum_{m=0}^{n} b_{m} b_{m n} .
$$

7. Analogue of descending power series expansion. The expansion

$$
1 /(x-t)=\sum_{n=0}^{\infty} U_{n}(t) v_{n}(x)
$$

is valid for $|t|<|x|$ and $|t|<\sigma$. Let $\Gamma_{1}$ be the circle $|x|=r_{1} \leqq \sigma$ and let $\Gamma_{2}$ be the circle $|t|=r_{2}<r_{1}$. Then if $g(x)$ is single-valued and analytic on and without $\Gamma_{2}$ and if $g(\infty)=0$, we have

where

$$
\begin{aligned}
g(x) & =\frac{1}{2 \pi i} \int_{\Gamma_{2}} \frac{g(t) d t}{(x-t)} \\
& =\sum_{n=0}^{\infty} a_{n}{ }^{\prime} v_{n}(x),
\end{aligned}
$$

$$
a_{n}{ }^{\prime}=\frac{1}{2 \pi i} \int_{\Gamma_{2}} U_{n}(t) g(t) d t,
$$

this expansion being valid for $|x|>r_{2}$ and converging uniformly for $|x| \geqq r_{1}$. Hence we have proved

ThEOREM III. Let $f(x)$ be single-valued and analytic on and without the circle $\Gamma_{2}$ with center at $x=0$ and radius $r_{2}<\sigma$. Then the expansion

$$
f(x)=f(\infty)+\sum_{n=0}^{\infty} a_{n}{ }^{\prime} v_{n}(x)
$$

where

$$
a_{n}{ }^{\prime}=\frac{1}{2 \pi i} \int_{\Gamma_{\mathbf{n}}} U_{n}(t)\{f(t)-f(\infty)\} d t,
$$

is valid for $|x|>r_{2}$ and converges uniformly for $|x| \geqq r_{1}>r_{2}$. 
8. Analogue of Laurent's series. Let $\Gamma_{2}$ and $C_{2}$ be the circles with centers at $x=0$ and radii $r_{2}$ and $R_{2}$ respectively, with $r_{2}<R_{2} \leqq \sigma$, and let $f(x)$ be single-valued and analytic on the boundary and interior of the ringshaped region $S$ defined by the inequalities

$$
0<r_{2} \leqq|x| \leqq R_{2} \leqq r \cdot
$$

Then if $x$ is any interior point of $S$, Laurent's theorem states that

$$
f(x)=\frac{1}{2 \pi i} \int_{\Gamma_{2}} \frac{f(t) d t}{(x-t)}+\frac{1}{2 \pi i} \int_{C_{2}} \frac{f(t) d t}{(t-x)} .
$$

Now in the first of these integrals

$$
|t|<|x|<\sigma
$$

so that here we may put

and in the second

$$
1 /(x-t)=\sum_{n=0}^{\infty} U_{n}(t) v_{n}(x)
$$

$$
|x|<|t| \leqq \sigma,
$$

so that in this integral we have

$$
1 /(t-x)=\sum_{n=0}^{\infty} v_{n}(t) U_{n}(x) .
$$

Therefore $f(x)$ has the expansion

$$
f(x)=\frac{1}{2 \pi i} \int_{\Gamma_{2}}\left\{\sum_{n=0}^{\infty} U_{n}(t) v_{n}(x)\right\} f(t) d t+\frac{1}{2 \pi i} \int_{C_{2}}\left\{\sum_{n=0}^{\infty} v_{n}(t) U_{n}(x)\right\} f(t) d t .
$$

From this last relation we may at once deduce the following theorem:

THEOREM IV. Let $f(x)$ be single-valued and analytic on the boundary and interior of the ring-shaped region $S$ defined by the inequalities

Then the expansion

$$
0<r_{2} \leqq|x| \leqq R_{2} \leqq \sigma .
$$

$$
f(x)=\sum_{n=0}^{\infty} a_{n} U_{n}(x)+\sum_{n=0}^{\infty} \alpha_{n} v_{n}(x)
$$

where

$$
a_{n}=\frac{1}{2 \pi i} \int_{C_{2}} v_{n}(t) f(t) d t
$$


and

$$
\alpha_{n}=\frac{1}{2 \pi i} \int_{\Gamma_{1}} U_{n}(t) f(t) d t
$$

is valid for every interior point of $S$ and converges uniformly when $x$ is in the region defined by the inequalities

$$
r_{2}<r_{1} \leqq|x| \leqq R_{1}<R_{2} .
$$

If the Laurent expansion of $f(x)$ in the region $S$ is

$$
f(x)=\sum_{n=0}^{\infty} b_{n} x^{n}+\sum_{n=1}^{\infty} \beta_{n} x^{-n},
$$

one readily finds that

and

$$
a_{n}=\sum_{m=0}^{n} b_{m} b_{m n}
$$

$$
\alpha_{n}=\beta_{n+1}+\sum_{m=1}^{\infty} c_{n m} \beta_{n+m+1}
$$

9. Orthogonality properties. We shall now obtain the following formulas :

$$
\begin{array}{ll}
\int_{D} U_{m}(x) U_{n}(x) d x=0 & (m=n \text { and } m \neq n), \\
\int_{D} v_{m}(x) v_{n}(x) d x=0 & (m=n \text { and } m \neq n), \\
\int_{D} U_{m}(x) v_{n}(x) d x=0 & (m \neq n), \\
\int_{D} U_{m}(x) v_{m}(x) d x=2 \pi i, &
\end{array}
$$

where the integration is taken in a positive direction along $D$, a closed curve which encircles the point $x=0$ once and lies within the circle $|x|=\sigma$. The first of these formulas follows immediately from the fact that $U_{m}(x)$ and $U_{n}(x)$ are analytic on and within $D$. The second one holds since the product $v_{m}(x) v_{n}(x)$ consists of terms in $x^{-2}$ and lower powers of $x$. The third relation is evident if $m>n$, since then there is no term in $x^{-1}$ in the product $U_{m}(x) v_{n}(x)$. If $m<n$ the coefficient of $x^{-1}$ in $U_{m}(x) v_{n}(x)$ is

$$
b_{m, m+\varepsilon}+b_{m+1, m+\varepsilon} c_{m 1}+b_{m+2, m+s} c_{m 2}+\cdots+b_{m+s, m+s} c_{m s},
$$

where $s$ has been put for $n-m$. To see that this coefficient vanishes, write 
the second of equations (11) for $s=1,2,3, \cdots, s$ and solve this system for $b_{m, m+c .}$ This gives the result

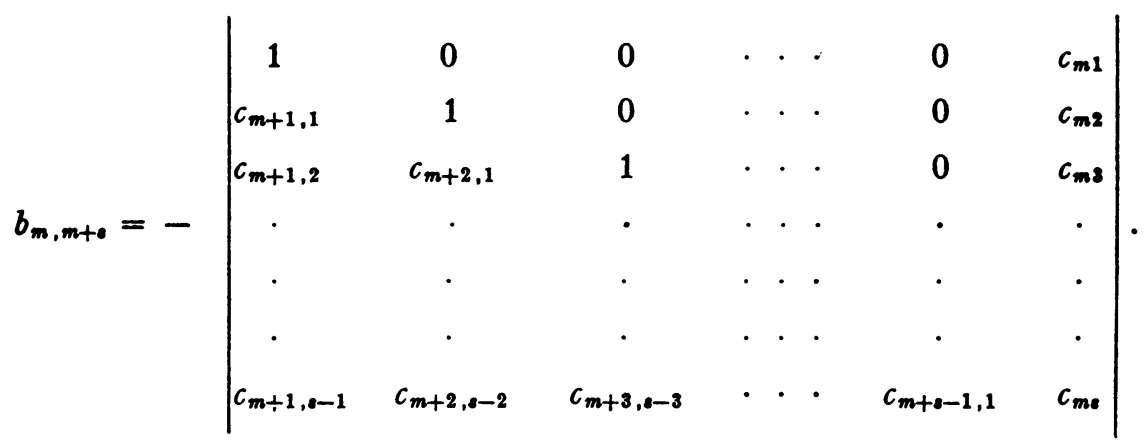

By expanding this determinant according to the elements of the last column, we readily obtain the relation

$$
b_{m, m+\varepsilon}=-\left(b_{m+1, m+{ }_{s}} c_{m 1}+b_{m+2, m+{ }_{s}} c_{m 2}+\cdots+b_{m+s, m+s} c_{m s}\right) .
$$

To verify the last of formulas (17) is it only necessary to note that the coefficient of $x^{-1}$ in $U_{m}(x) v_{m}(x)$ is unity.

By means of these formulas it is possible to obtain directly the coefficients in the expansions (14), (15), and (16). If, for example, we have an expansion of the type

$$
f(x)=\sum_{n=0}^{\infty} a_{n} U_{n}(x)+\sum_{n=0}^{\infty} \alpha_{n} v_{n}(x)
$$

and let $C$ be any curve in the ring-shaped region in which this expansion is valid and including the point $x=0$ once, then by multiplying both members of (16) by $U_{n}(x)$ and integrating term by term along $C$ with respect to $x$, we have

$$
\alpha_{n}=\frac{1}{2 \pi i} \int_{C} U_{n}(x) f(x) d x .
$$

Similarly, multiplying both members by $v_{n}(x)$ and integrating, we get

$$
a_{n}=\frac{1}{2 \pi i} \int_{C} v_{n}(x) f(x) d x .
$$

10. The case of linear coefficients. In the special case in which the coefficients $A_{0}(x), A_{1}(x), \cdots, A_{k}(x)$ are linear, equation (2) becomes

$$
A_{i}(x)=a_{i 0}+a_{i 1} x \quad(i=0,1,2, \cdots, k),
$$


while equation (4) is unchanged. The coefficients $c_{n s}$ and $b_{n, n+\varepsilon \text {, which }}$ may be readily calculated, are given by the equations

$$
\begin{aligned}
c_{n s} & =\prod_{i=0}^{s-1} \rho_{n+i} /\left(\lambda_{n+i+1}-\lambda_{n}\right), & & s>0, \\
b_{n n} & =1, & & s>0, \\
b_{n, n+s} & =\prod_{i=0}^{s-1} \rho_{n+i} /\left(\lambda_{n+i}-\lambda_{n+\varepsilon}\right), & &
\end{aligned}
$$

where

$$
\rho_{n}=\sum_{j=0}^{k} a_{j 1} q^{(k-j)(n+\nu)} .
$$

The radius $R$ of the circle within which the series

$$
x^{n+v}\left(1+\sum_{s=1}^{\infty} c_{n s} x^{s}\right)
$$

represents a solution of the $q$-difference equation is $\left|a_{00} / a_{01}\right|$ and it is not hard to show that the expansion (10), (11) is valid for $|x|\left\langle|q|{ }^{k}\left|a_{00} / a_{01}\right|\right.$, so that here the $\sigma$ of Theorems I, II, III, IV is equal to $|q| k\left|a_{00} / a_{01}\right|$; that is, it is equal to the radius of the circle of convergence of the power series expansion of $U_{n}(x)$.

In the case in which the functions $A_{0}(x), A_{1}(x), \cdots, A_{k}(x)$ are linear functions of $x$, the functions $v_{n}(t)$ satisfy a simple homogeneous $k$ th order $q$-difference equation with linear coefficients if the relation

$$
T \equiv a_{01} q^{k(\nu-1)}+a_{11} q^{(k-1)(\nu-1)}+a_{21} q^{(k-2)(\nu-1)}+\cdots+a_{k 1}=0
$$

is satisfied. For from the equation

$$
x^{\nu} /(t-x)=\sum_{n=0}^{\infty} v_{n}(t) u_{n}(x)
$$

and equation (1) it follows that

$$
\begin{aligned}
q^{k v} x^{r}\left(a_{00}+\right. & \left.a_{01} x\right) /\left(t-q^{k} x\right)+q^{(k-1)_{\nu} x^{\nu}}\left(a_{10}+a_{11} x\right) /\left(t-q^{k-1} x\right) \\
& +\cdots+x^{\nu}\left(a_{k 0}+a_{k 1} x\right) /(t-x)=-\sum_{n=0}^{\infty} \lambda_{n} v_{n}(t) u_{n}(x)
\end{aligned}
$$

or

$$
\begin{aligned}
& -T x^{\nu}+x^{\nu}\left(a_{00} q^{k(\nu-1)}+a_{01} t q^{k(\nu-2)}\right) /\left(t q^{-k}-x\right) \\
& +x^{\nu}\left(a_{10} q^{(k-1)(\nu-1)}+a_{11} t q^{(k-1)(\nu-2)}\right) /\left(t q^{-k+1}-x\right) \\
& +\cdots+x^{\nu}\left(a_{k 0}+a_{k 1} t\right) /(t-x)=-\sum_{n=0}^{\infty} \lambda_{n} v_{n}(t) u_{n}(x),
\end{aligned}
$$


whence

$$
\begin{aligned}
& \sum_{n=0}^{\infty} u_{n}(x)\left\{\left(a_{00} q^{k(\nu-1)}+a_{01} t q^{k(\nu-2)}\right) v_{n}\left(t / q^{k}\right)\right. \\
& +\left(a_{10} q^{(k-1)(\nu-1)}+a_{11} t q^{(k-1)(\nu-2)} v_{n}\left(t / q^{k-1}\right)\right. \\
& \left.+\cdots+\left(a_{k 0}+a_{k 1} t\right) v_{n}(t)\right\}=-\sum_{n=0}^{\infty} \lambda_{n} v_{n}(t) u_{n}(x) .
\end{aligned}
$$

Consequently the functions $v(t)$ satisfy the equation

$A_{0}{ }^{\prime}(t) v_{n}\left(t / q^{k}\right)+A_{1}{ }^{\prime}(t) v_{n}\left(t / q^{k-1}\right)+\cdots+A_{k-1}^{\prime}(t) v_{n}(t / q)+\left(\lambda_{n}+A_{k}{ }^{\prime}(t)\right) v_{n}(t)=0$, where

$$
A_{i}{ }^{\prime}(t)=a_{i 0} q^{(k-i)(\nu-1)}+a_{i 1} q^{(k-i)(\nu-2)} t \quad(i=0,1,2, \cdots, k) .
$$

If the relation $T=0$ is not satisfied, $v_{n}(t)$ evidently satisfies a non-homogeneous equation.

More generally, if the functions $A_{0}(x), A_{1}(x), \cdots, A_{k}(x)$ are polynomials, each of degree $d$ or less, $v_{n}(t)$ satisfies a homogeneous linear $q$ difference equation of order $k$ with coefficients $A_{i}^{\prime}(t)$, where $A_{i}^{\prime}(t)$ is a polynomial of the same degree as $A_{i}(x)$, provided that the $d(d+1) / 2$ equations

$$
\sum_{i=0}^{k} a_{i j} q^{(k-i)(\nu-\mu)}=0 \quad(j=1,2,3, \cdots, d ; \mu=1,2,3, \cdots, j)
$$

are satisfied. If some one of these equations fails to hold, $v_{n}(t)$ satisfies a non-homogeneous equation.

11. The problem when $|q|<1$. If, instead of supposing that $|q|>1$, we assume that $|q|<1$, the problem is essentially the same as before with the rôles of the points infinity and zero interchanged. If in equation (1) we replace $x$ by $1 / y$ and $q$ by $1 / p$ and put $A_{i}(1 / y)=B_{i}(y)$ and $u_{n}(1 / y)=w_{n}(y)$, we have

$$
\begin{aligned}
B_{0}(y) w_{n}\left(p^{k} y\right) & +B_{1}(y) w_{n}\left(p^{k-1} y\right) \\
& +\cdots+B_{k-1}(y) w_{n}(p y)+\left(\lambda_{n}+B_{k}(y)\right) w_{n}(y)=0,
\end{aligned}
$$

where $|p|<1$, the coefficients $B_{i}(y)$ are analytic at $y=\infty$, and $B_{0}(\infty) \neq 0$. Consequently, if we suppose that in equation (1) the coefficients $A_{i}(x)$ are analytic at $x=\infty, A_{0}(\infty)$ being different from zero, and that $|q|<1$, we may obtain expansions of arbitrary functions analytic at infinity in terms of solutions

$$
u_{-n}(x)=x^{-n-\nu}\left(1+\sum_{s=1}^{\infty} c_{-n, 8} x^{-s}\right),
$$


corresponding to the expansions of Theorems II, III, IV, and V. These expansions may be obtained directly by expanding $x^{-m-\rightarrow}$ in terms of the $u_{-n}(x)$, substituting these expansions in the identity

$$
x^{-\nu} /(x-t)=\sum_{m=0}^{\infty} t^{m} / x^{m+v+1},
$$

and applying Cauchy's theorem as before.

If the coefficients $A_{i}(x)$ are analytic at both zero and infinity, as, for example, when each function $A_{i}(x)$ is the quotient of one polynomial, $E_{i}(x)$, divided by another, $F_{i}(x)$, of the same degree, $F_{i}(0)$ being different from zero, there are expansions in both cases, $|q|<1$ and $|q|>1$.

III. EXPANSIONS OF ANALYTIC FUNCTIONS OF SEVERAL VARIABLES

12. The expansion theorems obtained in Section II may easily be extended to apply to functions of several variables. If $x_{1}, t_{1}$, and $x_{2}, t_{2}$ are two pairs of complex variables in the $x_{1}$ - and $x_{2}$-planes respectively, such that

$$
\begin{array}{lll}
\left|x_{1}\right| \leqq R_{1}, & \left|t_{1}\right| \geqq R_{1}{ }^{\prime}, & R_{1}<R_{1}{ }^{\prime} \leqq \sigma, \\
\left|x_{2}\right| \leqq R_{2}, & \left|t_{2}\right| \geqq R_{2}{ }^{\prime}, & R_{2}<R_{2}{ }^{\prime} \leqq \sigma,
\end{array}
$$

then by Theorem I the expansions

and

$$
1 /\left(t_{1}-x_{1}\right)=\sum_{n_{1}=0}^{\infty} v_{n_{1}}\left(t_{1}\right) U_{n_{1}}\left(x_{1}\right)
$$

$$
1 /\left(t_{2}-x_{2}\right)=\sum_{n_{2}=0}^{\infty} v_{n_{2}}\left(t_{2}\right) U_{n_{2}}\left(x_{2}\right)
$$

are valid and each converges uniformly with respect to both variables. Multiplying the two series together, we obtain their product

$$
\frac{1}{\left(t_{1}-x_{1}\right)} \frac{1}{\left(t_{2}-x_{2}\right)}=\sum_{n_{1}=0}^{\infty} \sum_{n_{2}=0}^{\infty} v_{n_{1}}\left(t_{1}\right) v_{n_{2}}\left(t_{2}\right) U_{n_{1}}\left(x_{1}\right) U_{n_{3}}\left(x_{2}\right),
$$

which is absolutely and uniformly convergent.

Let $C_{1}$ and $C_{2}$ be the contours formed by the circles $\left|x_{1}\right|=R_{1}{ }^{\prime}$ and $\left|x_{2}\right|=R_{2}^{\prime}$ and let $f\left(x_{1}, x_{2}\right)$ be a single-valued analytic function of the two variables $x_{1}$ and $x_{2}$ when $x_{1}$ is on and within $C_{1}$ and $x_{2}$ is on and within $C_{2}$. Then we have that 


$$
\begin{aligned}
f\left(x_{1}, x_{2}\right) & =\left(\frac{1}{2 \pi i}\right)^{2} \int_{C_{1}} \int_{C_{2}} \frac{f\left(t_{1}, t_{2}\right)}{\left(t_{1}-x_{1}\right)\left(t_{2}-x_{2}\right)} d t_{2} d t_{1} \\
& =\left(\frac{1}{2 \pi i}\right)^{2} \int_{C_{1}} \int_{C_{2}}\left\{\sum_{n_{1}=0}^{\infty} \sum_{n_{2}=0}^{\infty} v_{n_{1}}\left(t_{1}\right) v_{n_{2}}\left(t_{2}\right) U_{n_{1}}\left(x_{1}\right) U_{n_{2}}\left(x_{2}\right)\right\} f\left(t_{1}, t_{2}\right) d t_{2} d t_{1}, \\
& =\sum_{n_{1}=0}^{\infty} \sum_{n_{2}=0}^{\infty} a_{n_{12}} U_{n_{1}}\left(x_{1}\right) U_{n_{2}}\left(x_{2}\right),
\end{aligned}
$$

where

$$
a_{n_{1 n 2}}=\left(\frac{1}{2 \pi i}\right)^{2} \int_{C_{1}} \int_{C_{2}} v_{n_{1}}\left(t_{1}\right) v_{n_{2}}\left(t_{2}\right) f\left(t_{1}, t_{2}\right) d t_{2} d t_{1} .
$$

This expansion is valid and converges uniformly with respect to $x_{1}$ and $x_{2}$ when $\left|x_{1}\right| \leqq R_{1}$ and $\left|x_{2}\right| \leqq R_{2}$.

In precisely the same manner we may prove the following theorem:

THEOREM V. Let $f\left(x_{1}, x_{2}, \cdots, x_{\lambda}\right)$ be a function of $x_{1}, x_{2}, \cdots, x_{\lambda}$ which is single-valued and analytic when $x_{1}, x_{2}, \cdots, x_{\lambda}$ are on and within the respective contours $C_{1}, C_{2}, \cdots, C_{\lambda}$ formed by the circles $\left|x_{1}\right|=R_{1}^{\prime} \leqq \sigma$, $\left|x_{2}\right|=R_{2}^{\prime} \leqq \sigma, \cdots,\left|x_{\lambda}\right|=R_{\lambda}{ }^{\prime} \leqq \sigma$. Then the expansion

$$
f\left(x_{1}, x_{2}, \cdots, x_{\lambda}\right)=\sum_{n_{1}=0}^{\infty} \sum_{n_{2}=0}^{\infty} \cdots \sum_{n_{\lambda}=0}^{\infty} a_{n_{1} n_{2} \ldots n_{\lambda}} U_{n_{1}}\left(x_{1}\right) U_{n_{2}}\left(x_{2}\right) \cdots U_{n_{\lambda}}\left(x_{\lambda}\right),
$$

where

$a_{n_{1} n_{2} \ldots n_{\lambda}}=\left(\frac{1}{2 \pi i}\right)^{\lambda} \int_{C_{1}} \int_{C_{2}} \cdots \int_{C_{\lambda}} v_{n_{1}}\left(t_{1}\right) v_{n_{2}}\left(t_{2}\right) \cdots v_{n_{\lambda}}\left(t_{\lambda}\right) f\left(t_{1}, t_{2}, \cdots, t_{\lambda}\right) d t_{\lambda} \cdots d t_{1}$, is valid for $\left|x_{1}\right|<R_{1}{ }^{\prime},\left|x_{2}\right|<R_{2}^{\prime}, \cdots,\left|x_{\lambda}\right|<R_{\lambda}{ }^{\prime}$ and converges uniformly for

$$
\left|x_{1}\right| \leqq R_{1}<R_{1}{ }^{\prime},\left|x_{2}\right| \leqq R_{2}<R_{2}{ }^{\prime}, \cdots,\left|x_{\lambda}\right| \leqq R_{\lambda}<R_{\lambda}{ }^{\prime} .
$$

Evidently Theorems III and IV may be generalized in like manner.

\section{UNIVERSITY OF ILLINOIS,}

$$
\text { Urbana, ILL. }
$$

